\title{
An unusual infection in a patient with peripherally inserted central catheter
}

BY YANYAN ZHOU, GUYI WANG, YOUDI LV, HAIYUN DONG, JINXIU LI, JIANJUN TANG

\section{Abstract}

A peripherally inserted central catheter (PICC) is widely used in transfusion therapy and for monitoring many kinds of diseases, especially in critically ill patients. Compared with other catheters, it has a lower risk of catheter-related bloodstream infections. Aeromonas Hydrophila (AH) is a kind of opportunistic pathogen, vibrionaceae aeromonas, and gramnegative brevibacterium, widely distributed in nature, in all kinds of body fluid. It usually causes gastrointestinal infections, and rarely causes Aeromonas septicemia. To date, there has been no report of a PICCrelated $\mathrm{AH}$ infection. We report the case of a 40-year-old female with breast cancer, who suffered post-op. severe sepsis and double lower limb cellulitis with multiple organ failure. All of this was due to AH invading the blood through the PICC.

Key words: Aeromonas Hydrophila, peripherally inserted central catheter, sepsis, multiple organ failure

\section{Introduction}


Aeromonas Hydrophila (AH) is a Gram-negative, aquatic bacterium which causes infection in both human beings and animals. (1) Less than 5 cases of catheter-related infections due to $\mathrm{AH}$ have been reported in the medical literature, and most of the reports involved infections that were not very serious. However, we present a case of severe sepsis and cellulitis of both lower limbs due to $\mathrm{AH}$ in a breast cancer patient receiving postoperative chemotherapy. Despite aggressive treatment, this terrible infection caused multiple organ failure. Our aim is to highlight the threat of $\mathrm{AH}$ to patients with an indwelling peripherally inserted central catheter (PICC).

\section{Case report}

A 40-year-old female farmer was admitted to our hospital with a fever and left lower limb pain after flushing the PICC by herself 2 days ago. She had received 5 cycles of postoperative chemotherapy (docetaxel 120mg, pirarubicin 6omg, cyclophosphamide 8oomg for 1 day) for left breast invasive carcinoma, over the past 4 months. The PICC was inserted via ultrasound-guided venepuncture of the right brachial vein. Her vital signs were normal except for a high body temperature $\left(38.6^{\circ} \mathrm{C}\right)$. Her left breast was absent, and left lower limb was obviously swollen, with thinning skin, local visible blister formation and tenderness. No infection at the PICC puncture point was observed. Lab tests revealed leukocytosis (white blood cell 36.6×109/1, with 95.3\% neutrophils), severe renal impairment (creatinine 586.9umol/1), and negative viral hepatitis serology.. The patient had no history of chronic disease.

We removed the catheter, and cultured the tip and percutaneous peripheral blood for microbiological examination (two sets each in aerobic and anaerobic bottles ; Becton, Dickinson and Company). However, her condition deteriorated after admission with apparent chills and fever $\left(41.6^{\circ} \mathrm{C}\right)$, apathetic facial expressions, hypotension, tachycardia, oliguria, and altered consciousness. Her left lower limb showed cyanopathy, local bursting of blisters, with a reddish, transparent exudate, which was diagnosed as cellulitis by the consultant dermatologist. , We then swabbed the tissue to perform a microbiological examination. The empirical intravenous antibiotic imipenem/cilastatin 
was initiated (50omg every 8h).

The patient was transferred to the Intensive Care Unit with multiple organ failure and double lower limb cellulitis on day 3. Her lab tests showed leukocytosis (white blood cell $16.9 \times 10^{9} / 1$ with $95.84 \%$ neutrophils), and thrombocytopenia ( $25 \times 10^{9} / 1$ ), severe liver and kidney damage ( alanine aminotransferase $1809.4 \mathrm{u} / \mathrm{l}$, aspartate aminotransferase $2914.2 \mathrm{u} / \mathrm{l}$, total bilirubin $102.6 \mathrm{umol} / \mathrm{l}$, direct bilirubin 89.0umol/l, urea nitrogen 30.10umol/l, creatinine 409.9umol/l), coagulation dysfunction (prothrombin time 68.9s, activated partial thromboplastin time 128.5s, international normalized ratio 8.09, DDimer $31.58 \mathrm{ug} / \mathrm{ml}$ ), and obvious tissue oxygen hypoperfusion (myoglobin 10279.5u/l, blood lactate $3.1 \mathrm{mmol} / \mathrm{l})$, high procalcitonin $(55.6 \mathrm{ong} / \mathrm{ml}$ ). We give her blood products transfusion, analgesia, liver protection treatments, and continuous renal replacement therapy.

On day 4, the strain isolated from the percutaneous peripheral blood specimens (collected on admission) was identified as AH, which was susceptible to amikacin, aztreonam, ceftazidime, ciprofloxacin and meropenem, but was resistant to imipenem. The pathogen and drug susceptibility testing of the PICC tip was the same as the above. Therefore, the intravenous antibiotic was changed to meropenem (1000mg every 8h).

Unfortunately, the patient remained febrile, with high infection parameters (figure 1). She experienced a variety of bacterial and fungal infections, including Coagulase-negative staphylococcus, Acinetobacter baumannii (generic drug resistance), Pseudomonas aeruginosa (multiple drug resistant), and Tropical candida. After 33 days of anti-infection treatment, the patient was finally discharged from hospital.

\section{Discussion}

Because of its particularly low incidence of catheter related bloodstream infections, PIC lines have been widely used globally. (2) AH is an opportunistic pathogen, vibrionaceae aeromonas, and gram-negative brevibacterium, which is widely distributed in nature, in all kinds of body 
fluid. AH produces exotoxins of highly toxic nature, such as: hemotoxin, histotoxin, necrotoxin, enterotoxin and protease. All 7 patients, reported by Morinaga $Y$, over 10 years were infections occurring in the summer and fall season, and immunosuppressive state and eating raw fish or shellfish were the common causes. (3) Venous access devices ( VADs) related infections caused by $\mathrm{AH}$ have only been reported in a dialysis catheter. (4)

There were only several cases of catheter-related infections caused by AH that have been reported in the past 5years, mostly in central venous catheters (dialysis catheter) or peritoneal dialysis catheters (table 1). (47) The catheter indwelling times ranged from several days to several years, and patients were a combination of immunosuppressive state or not. The symptoms varied according to invasive positions: hemodialysis patients often had a cough, respiratory distress and shock as the main symptoms, $(4,5)$ whereas peritoneal dialysis (PD) patients had nausea, vomiting, abdominal pain, abdominal muscle tension in the abdominal cavity infection. $(6,7)$ Antibiotic administration times were between 14 and 21 days, the prognoses were relatively good, with no other infections.

This case is the first one about AH infection in patients through a PICC. Both of the primary microbiological results of percutaneous peripheral blood and PICC tip revealed AH infection. These indicated that PICC may be the infection source. Considering the patient had a history of flushing the catheter by herself before hospitalization, we speculate that the fluid for PICC infusion or some other devices were contaminated with AH. The severity of the infection and the time of hospitalization are both more than in previous similar cases.

In conclusion, prevention is the key to avoiding PICC related sepsis caused by AH. In China, these are a lot of outpatients carrying VADs, especially cancer patients and other chronically illn patients. PICC is one of the most used VADs. It suggests doctors should be alerted to PICC related sepsis caused by AH and strengthen the awareness of the importance of hand hygiene and sterile conditions.

\section{Acknowledgement}


We thank the staff from the clinical bacteriology laboratory for their critical review and helpful suggestions. We also thank the patient for providing permission to publish her information.

\section{References}

1. Chang CY, Thompson H, Rodman N, Bylander J, Thomas J. Pathogenic analysis of Aeromonas hydrophila septicemia. Ann Clin Lab Science 1997;27:254-59.

2. $\underline{\text { Cotogni P }}, \underline{\text { Pittiruti M}}, \underline{\text { Barbero C }}, \underline{\text { Monge T }}, \underline{\text { Palmo A }}$, Boggio Bertinet D.Catheter-related complications in cancer patients on home parenteral nutrition: a prospective study of over 51,00o catheter days. JPEN J Parenter Enteral Nutr 2013;37:375-83.

3. Morinaga Y,$\underline{\text { Yanagihara K }}$, Araki N,$\underline{\text { Harada Y }}$, $\underline{\text { Yamada K }}, \underline{\text { Akamatsu }}$ $\underline{\mathrm{N}}$, et al. Clinical characteristics of seven patients with Aeromonas septicemia in a Japanese hospital. Tohoku J Exp Med 2011;225:81-4.

4. Khalil MA, Rehman A, Kashif WU, Rangasami M, Tan J. A rare case of Aeromonas hydrophila catheter related sepsis in a patient with chronic kidney disease receiving steroids and dialysis: a case report and review of Aeromonas infections in chronic kidney disease patients. Case Rep Nephrol 2013;2013:735194.

5. Zhou Z, Guo D. Catheter-related bacteremia caused by Aeromonas hydrophila in a hemodialysis patient. Infect Control Hosp Epidemiol 2013;34:765-6.

6. $\underline{\text { Sahin I1 }}$ Barut HS.Quinolone-resistant Aeromonas hydrophila peritonitis in a CAPD patient. Clin Nephrol 2010;73:241-3.

7. Liakopoulos V, Arampatzis S, Kourti P, Tsolkas T, Zarogiannis $\underline{\mathrm{S}}$, Eleftheriadis T, et al. Aeromonas hydrophila as a causative organism in peritoneal dialysis-related peritonitis: case report and review of the literature. Clin Nephrol 2011;75:65-8. 


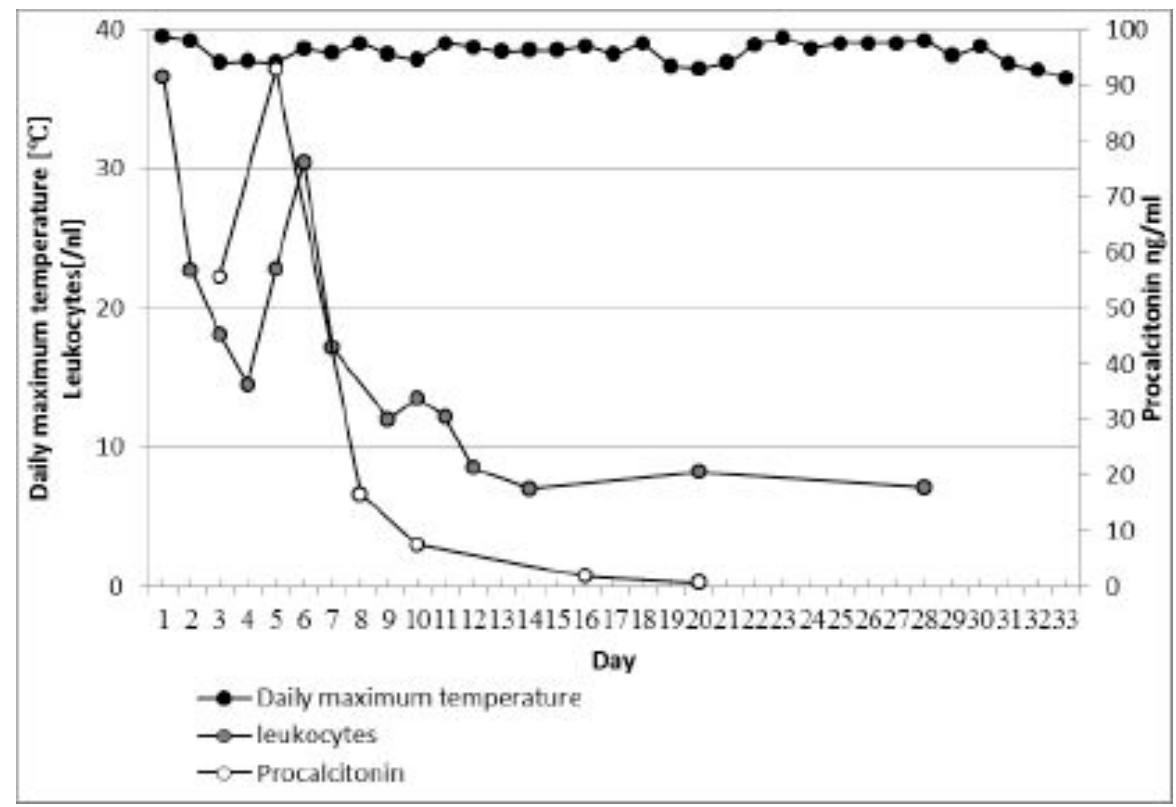

Figure 1. Infection parameters during the hospital stay: daily maximum temperature, leukocytes, procalcitonin.

Table 1. Case reports of catheter-related infections caused by Aeromonas Hydrophila.

\begin{tabular}{|c|c|c|c|}
\hline Author & $\begin{array}{l}\text { Catheter type } \\
\text { and } \\
\text { indwelling } \\
\text { time }\end{array}$ & Co-morbidity & $\begin{array}{l}\text { Antibiotics- } \\
\text { using } \\
\text { time(days) }\end{array}$ \\
\hline
\end{tabular}

Zhou Z

Dialysis

Diabetic

(5)

catheter,16days

nephropathy

21

Khalil Dialysis

MA (4)

catheter,12days

IgA nephropathy

14

Sahin I

Peritoneal

Chronic

dialysis

catheter,5years

glomerulonephritis

14

Liakopoulos $\underline{\mathrm{V} b}(7)$

Peritoneal

Systemic

dialysis

lupus erythematosus

21

Present case

PICC,4months

Postoperative

chemotherapy due to

breast cancer 
PICC, peripheral inserted central catheter.

Corresponding Address

Jianjun Tang

Intensive Care Unit

The Second Xiangya Hospital

Central South University, Changsha, Hunan, 410011, China

Phone: +8673185295170 .

E-mail address: tom200210@163.com

Article printed from Signa Vitae: http://www.signavitae.com

URL to article: http://www.signavitae.com/2015/12/an-unusualinfection-in-a-patient-with-peripherally-inserted-centralcatheter/

Copyright (C) 2015 Signa Vitae. All rights reserved. 